\title{
Sistema para la generación automática de mapas de rendimiento. Aplicación en la agricultura de precisión
}

\author{
System for the automatic generation of yield mapping with application \\ in precision farming \\ Carlos Lago González ; Juan Carlos Sepúlveda Peña ${ }^{2}$; Rogelio Barroso Abreu; \\ Félix Óscar Fernández Peña ${ }^{4}$; Francisco Maciá Pérez; ; Javier Lorenzo $0^{6}$
}

\begin{abstract}
RESUMEN
La creciente preocupación medioambiental y la necesidad de producir alimentos de calidad de una manera sostenible y respetuosa con el entorno ubican al sector agroalimentario en el punto de mira de la sociedad. El empleo de la tecnología del Sistema de Posicionamiento Global (GPS, siglas en inglés) en el ámbito civil ha fomentado el surgimiento de nuevas fuentes de desarrollo, entre las que se puede mencionar la Agricultura, y muy particularmente la Agricultura de Precisión. Muy brevemente se puede definir como la posibilidad de aplicar tratamientos distintos a escala local de un cultivo y obtener una mayor producción con un menor costo. El rendimiento agrícola resume el resultado de todo el ciclo de producción de un cultivo, por lo que su censado y mapeo brindan la posibilidad de conocer cómo fue su variabilidad sobre el terreno y ofrecen la posibilidad de manejarlo. Con la elaboración de un mapa de rendimiento del cultivo y una vez obtenido el rendimiento de cada punto se le puede aplicar una dosis variable de pesticidas, abonos, fertilizantes, etc., contribuyendo a minimizar el costo de la producción y a lograr un mejor equilibro ambiental.
\end{abstract}

Palabras clave: Agricultura de Precisión, Mapas de Rendimiento, Mapas de Aplicación, Sistemas de Posicionamiento Global, Sistemas de Información Geográfica, Interpolación, Imagen.

\begin{abstract}
The growing environmental concern and the necessity to produce quality foods in a sustainable way which respects the environment have put the agricultural sector under the scrutiny of society. The employment of the Global Positioning System (GPS) technology in the civilian sector has sparked the emergence of new technological applications, in agriculture and very particularly in Precision Agriculture, considered to be among the 10 most important sciences for the XXI century. Precision agriculture refers to the application of different treatments at the local cultivation scale, which achieves greater production and better product quality with lower costs. Agricultural yield is the best indicator of the entire cultivation cycle of a crop; its census and mapping allows the variability of the area to be determined and managed. To achieve this it is necessary to produce a yield map of the cultivated area; once the yield of each point (understood to be a very small area inside a field, for example a cell of a square meter) is obtained, variable doses of pesticides, fertilizers, etc. may be applied, contributing to minimize production costs and to achieve a better environmental balance.
\end{abstract} Report.

Key words: Precision Agriculture, Yield Mapping, Application Mapping, GPS, Productivity Report, Profitability

1 Ingeniero en Informática. Máster en Ciencias en Informática Aplicada. Profesor Asistente del Instituto Superior Politécnico José A. Echeverría. Calle 114 No 11901 e/119 y 127. Marianao, C. La Habana, Cuba. CP 19390. Tel. (53-7) 266 3801. E-mail: clago@ceis.cujae.edu.cu

2 Ingeniero en Control Automático, Master en Ciencias, Profesor Auxiliar del Instituto Superior Politécnico José A. Echeverría. Calle 114 N $^{\circ} 11901$ e/119 y 127. Marianao, C. La Habana, Cuba. CP 19390. Tel. (53-7) 266-3801. E-mail: JCarlos@ ceis. cujae.edu.cu

3 Ingeniero Informático, profesor adiestrado del Instituto Superior Politécnico José A. Echeverría. Calle $114 \mathrm{~N}^{\circ} 11901$ e/119 y 127. Marianao, C. La Habana, Cuba. CP 19390. Tel. (53-7) 26064 18. E-mail: rbarroso@ ceis.cujae.edu.cu

4 Ingeniero en Informática, Doctor en Ciencias, Máster en Ciencias, Profesor Auxiliar del Instituto Superior Politécnico José A. Echeverría. Calle 114 No 11901 e/119 y 127. Marianao, C. La Habana, Cuba. CP 19390. Tel. (53-7) 266-3801. E-mail: felix@ceis.cujae.edu.cu

5 Dr. en Ciencias, Profesor Titular de la Universidad de Alicante y Jefe del Departamento de Tecnología Informática y Computación. Campus de Sant Vicent del Raspeig, AP 99 E-03080, Alicante, España. Tel. 96590 3463. E-mail: pmacia@ dtic.ua.es

6 Técnico Medio en Informática. Instituto Superior Politécnico José A. Echeverría. Calle 114 No 11901 e/119 y 127. Marianao, C. La Habana, Cuba. CP 19390. Tel. (53-7) 266 3801. E-mail: Javier@ ceis.cujae.edu.cu 


\section{Introducción}

En la década del 70 el Departamento de Defensa Americano comenzó el proyecto para el lanzamiento de satélites geoestacionarios alrededor de la Tierra, con el propósito de localizar objetivos militares de forma exacta y rápida. A este proyecto se le denominó Sistema de Posicionamiento Global o GPS, aunque su nombre correcto es NAVSTARGPS (versión mejorada del sistema TRANSIT, NAVigation Satellite Timing And Ranging).

El principal objetivo del GPS cuando se creó era guiar proyectiles desde plataformas móviles hasta objetivos de países enemigos. Sin embargo, hoy en día se aplica a multitud de actividades civiles, tales como la Agricultura.

El GPS está dividido por tres segmentos [1]:

- Segmento Espacial.

- Segmento de Control.

- Segmento Usuario.

El GPS es utilizado en múltiples campos como la geodesia, geofísica, geodinámica, astronomía, meteorología, cartografía o topografía, y en un sinfín de ciencias más. También se utiliza en la navegación marina, aérea o terrestre, en la sincronización del tiempo, para controlar flotas y maquinarias, en la localización automática de vehículos o en la exploración y en los deportes de aventura.

\section{Aplicación del GPS}

Navegación: Las aplicaciones más extendidas del GPS son en el terreno de la navegación aérea, terrestre y marítima [2].

Cartografía - Topografía: La tecnología digital del GPS permite confeccionar mapas geográficos mucho más precisos, mejorando los que había hasta ahora [2].

Investigación: El GPS es un instrumento científico de precisión, permite monitorear numerosos fenómenos como los movimientos de la corteza terrestre o las migraciones de muchas especies animales [2]. Han surgido nuevas materias de investigación que dependen totalmente del GPS, como lo es la Agricultura de Precisión. La Agricultura de Precisión constituye un valioso instrumento para diagnosticar con exactitud problemas de la producción agrícola, adoptar decisiones y obtener respuestas satisfactorias en los índices de rendimiento agrícola. Prácticamente consiste en actuar hasta con el más mínimo detalle en el sitio adecuado y en el momento oportuno, a partir de las novedades científicas que ofrecen la informática y la tecnología [3].

\section{Agricultura de precisión}

\subsection{Antecedentes}

La Agricultura de Precisión (AP) es un concepto agronómico de gestión de parcelas agrícolas, basado en la existencia de variabilidad en campo. Requiere el uso de las tecnologías de Sistemas de Posicionamiento Global (GPS), sensores, satélites e imágenes aéreas junto con Sistemas de Información Geográfica (SIG) para estimar, evaluar y entender dichas variaciones. La información recolectada puede ser usada para evaluar con mayor precisión la densidad óptima de siembra, estimar fertilizantes y otras entradas necesarias, y predecir con más exactitud la producción de los cultivos [4].

Desde los inicios, la agricultura y, con ella el agricultor, ha ido modificando sus hábitos en el sentido de ir adaptándose a las necesidades del momento y teniendo claros siempre los objetivos. Las bases de la AP-variabilidad espacial y temporal del suelo y de los factores que afectan al cultivoson aspectos ya tenidos en cuenta a lo largo de la historia de la agricultura. Una de las primeras referencias bibliográficas que se puede citar se encuentra en la Biblia, en la parábola del sembrador (Mateo, 13 v 8). Ya en este caso se pusieron de manifiesto las consecuencias de la variabilidad espacial en los rendimientos del cultivo. Otra cosa es que las disponibilidades de materiales hagan esta consecución de objetivos más o menos complicada y difícil. Sin embargo, aspectos como la utilización de sembradoras de precisión, la modificación de las dosis de nitrógeno, de forma visual, en función del conocimiento previo de los cultivos, el ajuste de las dosis de semillas en función del tipo de suelo, la variación de los volúmenes de fitosanitarios basada en las necesidades puntuales, son en sí mismas una AP. Por lo tanto, más que hablar de un concepto nuevo y revolucionario, se hablará de la AP como una serie de elementos y sistemas que permiten mejorar, facilitar y automatizar todas aquellas operaciones que de forma más o menos habitual se vienen ya practicando [4]. 
La idea del Manejo Sitio Específico (MSE, área de mayor desarrollo dentro de la AP) es hacer lo correcto en el lugar adecuado y en el momento oportuno. Esta idea es tan vieja como la agricultura, pero durante la mecanización de la agricultura en el siglo XX hubo una gran motivación económica para tratar grandes extensiones de tierra con prácticas agronómicas uniformes. La AP proporciona una forma de automatizar el manejo sitio-específico usando la informática, por lo tanto haciendo que el manejo sitio-específico pudiera tener una aplicación práctica en la agricultura. La AP incluye todas las prácticas de producción agrícola que usan la informática para ajustar el uso de insumos de modo tal que permita obtener el producto deseado, o para monitorear dicho resultado, por ejemplo, la aplicación con Dosis Variable (DV), los monitores de rendimiento, los sensores, etc.[5].

La aplicación de conceptos de AP usualmente se considera relativa a la agricultura sostenible o sustentable. Esta pretende evitar la aplicación de las mismas prácticas a un cultivo, sin tener en cuenta las condiciones locales de suelo y clima y puede ayudar a evaluar situaciones locales de enfermedad [6]. Es la que, en el largo plazo, mejora la calidad del medio ambiente y de los recursos naturales de los que depende la agricultura; satisface las necesidades básicas de alimentación humana y de fibras; es económicamente viable; y mejora la calidad de vida de los productores y de la sociedad en general [5].

La agricultura no puede ser sostenible si los productores usan prácticas que no son socialmente aceptables o que no son rentables. Si se acepta que es inevitable usar algunos insumos externos en la producción agropecuaria, la AP puede ayudar a manejar esos insumos de forma tal que ayude a conservar el medio ambiente y aumentar la rentabilidad. Usando el conocimiento sitio-específico, la AP puede afinar las dosis de fertilizantes, semillas y otros agroquímicos de acuerdo al tipo de suelo y otras condiciones [5].

\section{2. ¿Qué es Agricultura de Precisión?}

Esta filosofía propone atender en forma diferenciada los factores de producción de acuerdo a las características específicas de cada sitio, con el fin de maximizar la eficiencia en el uso de los recursos, y minimizar los efectos de contaminación, usando como unidad de manejo el área más pequeña para la cual se cuenta con información de respaldo.
Metodológicamente implica la incorporación de las herramientas tecnológicas disponibles en la actualidad, especialmente aquellas que contemplan la referenciación geográfica de sitios vía satélite, unidos a bases de datos de información de esos sitios concretos, como sustento sobre la cual se apoyan las decisiones para el manejo. El proceso necesita la recolección de información en cada sitio, su ordenamiento y análisis y, finalmente, la diagramación de las estrategias para atender las limitantes a nivel de sitio. El manejo de los cultivos por lotes o secciones, es una tendencia que viene fortaleciéndose gradualmente en la mayoría de las actividades agrícolas. A ella se suma esta nueva corriente que carga al concepto con un fuerte componente tecnológico en la recolección y manejo de la información [7].

Lowenberg-DeBoer y Swinton (1997) definen el MSE como el "control y monitoreo electrónico aplicado a la recolección de datos, procesamiento de la información y apoyo para la toma de decisiones, para la ubicación temporal y espacial de insumos en la producción de cultivos".

La AP tiene el potencial de proporcionar a los productores modernas herramientas para manejar esos insumos que tienen que ser importados al campo. En lugar de aplicar fertilizantes o pesticidas indiscriminadamente en dosis uniformes sobre grandes áreas, la AP permite a los productores "afinar la puntería" con las aplicaciones de estas sustancias químicas. En cierto sentido, la AP sustituye algunos insumos físicos externos por la información y el conocimiento, acercando potencialmente el campo al ideal del balance biológico. Por supuesto, la tecnología informática y el conocimiento que hacen que la AP funcione, también son insumos externos. La esperanza que se pone en la AP es que sea menos desequilibradora de los sistemas naturales que los insumos físicos aplicados indiscriminadamente [5].

Por otro lado, el empleo del GPS permite que los agricultores puedan recopilar datos sobre sus terrenos de cultivo, ya sea durante la cosecha o previamente a ella, de tal manera que hoy por hoy los cultivos ya no han de ser necesariamente tratados como una superficie de terreno de características homogéneas, sino que pueden ser tratados acorde con sus características espaciales. Es decir, se ha pasado de trabajar en kilómetros cuadrados a trabajar en metros cuadrados. Esto se ve traducido en una mejor aplicación de pesticidas, semillas, riego, 
etc., todo lo cual conlleva un sustancial ahorro en costos variables de producción que, en su totalidad, compensan el gasto derivado del empleo de estas nuevas tecnologías [8].
A continuación se muestra (Figura 1) la relación de todos los elementos que integran la AP, así como los resultados esperados: la aplicación de las tecnologías y las ventajas de sus aplicaciones.

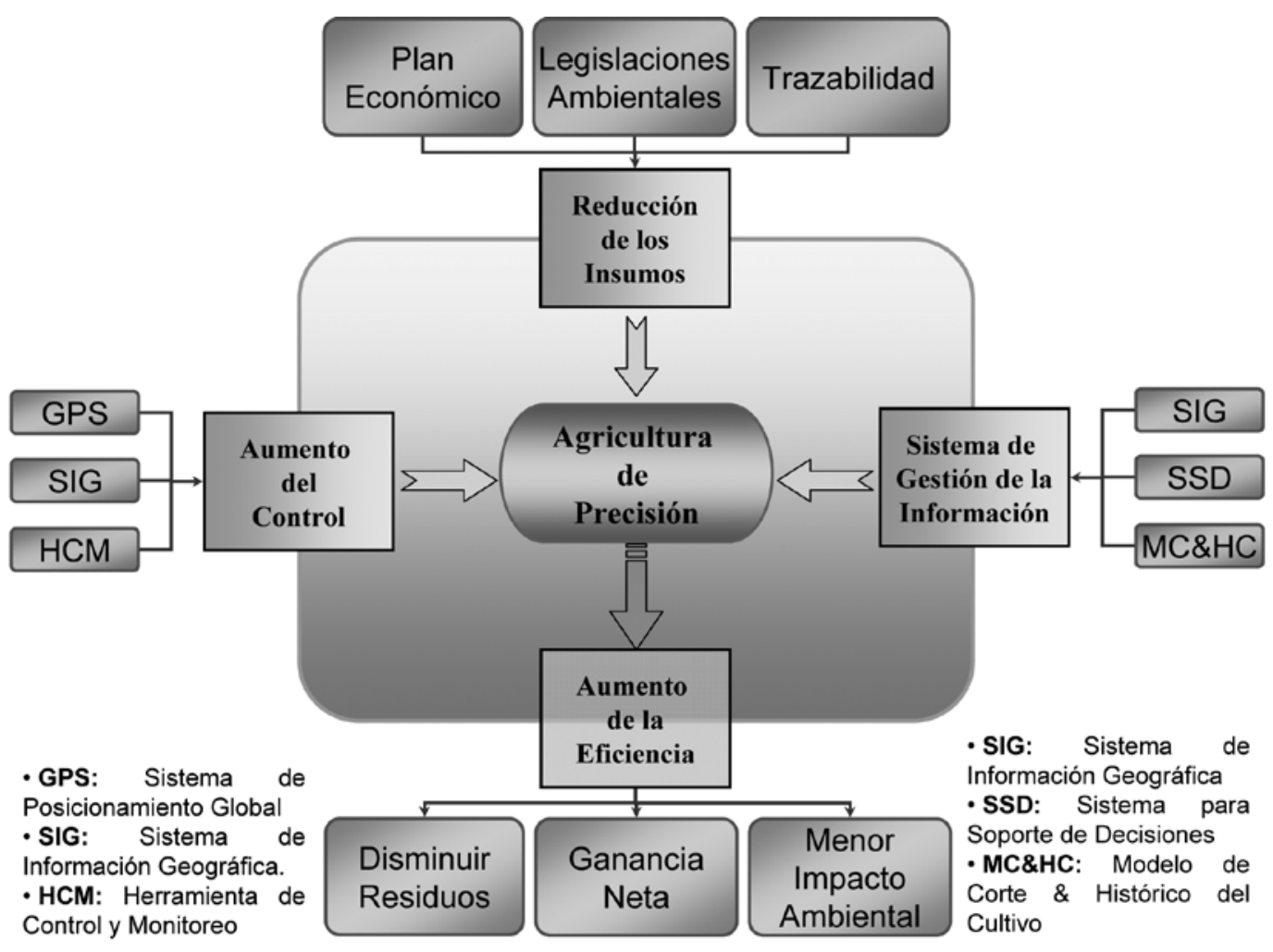

Figura 1. Interacción entre varios elementos que integran la AP [8].

La AP reconoce que la producción agrícola depende del suelo, clima, manejo pasado y varía en el espacio y en el tiempo. Por lo tanto las decisiones de manejo varían en forma sitio y tiempo específica, y no rígidamente programados como existen en la realidad [9].

El área de mayor desarrollo dentro de la Agricultura de Precisión es el MSE también llamado tecnología de Dosis Variable (DV) y corresponde a la aplicación variable de fertilizantes de acuerdo al nivel de fertilidad de cada sector de manejo homogéneo dentro del lote [9].

Esto significa que no se trabaja necesariamente con una sola dosis de fertilizante, sino que tantas dosis como áreas significativamente homogéneas existan en la explotación [9].

Sin embargo, existen otras áreas dentro del MSE, lotes como control sitio específico de malezas, insectos y enfermedades, aplicación variable de plaguicidas y densidad variable de semillas de acuerdo al potencial productivo del suelo [9].

Las prácticas MSE son recomendables para lotes donde existe una alta variabilidad de los factores de producción (fertilidad, malezas, agua, etc.) y los rendimientos varían en función de ellos [9].

La DV podría utilizarse con éxito cuando [9]:

- Los lotes presentan variabilidad del suelo que afecta el rendimiento y los rendimientos varían efectivamente en respuesta a la variación de las propiedades del suelo.

- Bajo estas condiciones se pueden aplicar manejos diferenciales dentro del sitio en términos de fertilización en lugar del tradicional promedio utilizado en la actualidad. 


\section{Sistema para la generación de los mapas de rendimiento}

\subsection{Mapas de Rendimiento}

Los Mapas de Rendimiento (MR) son imágenes georreferenciadas con una escala de colores que indican el rendimiento de un punto en específico. Por lo general, son desarrollados por científicos y especialistas de la Agricultura, donde los interesados (agricultores, cosechadores y productores) deben pagar un precio alto para obtenerlos. Los MR son entradas para el proceso de aplicación de Dosis Variable (DV) de los distintos químicos que necesita un cultivo (fertilizantes, herbicidas, riegos, etc.). Las cosechadoras, fertilizadoras, y otras maquinarias, necesitan de la instalación de un Computador de Abordo para el control y monitoreo, también es válido el uso de sensores de flujos para medir y registrar el rendimiento puntual (Figura 2).

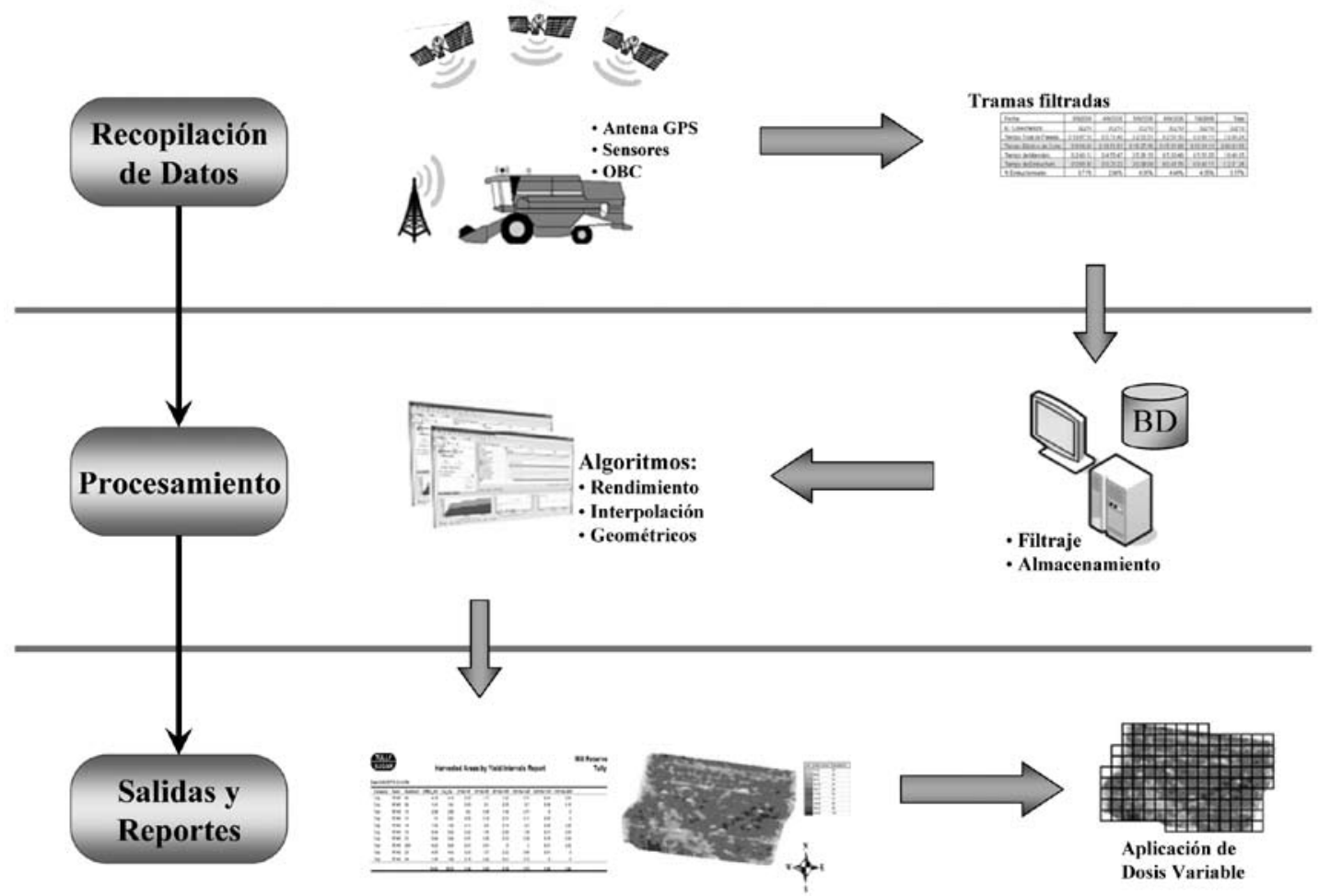

Figura 2. Proceso para generar Mapas de Rendimiento.

La propuesta que se presenta en este trabajo es un sistema automatizado para la generación de MR de forma sencilla y asequible para cualquier usuario y que no quede en manos solamente de los científicos y especialistas. La salida de este sistema será una imagen georreferenciada con varios ficheros asociados con ciertas particularidades como lo son:

Fichero para la exportación a un Sistema de Información Geográfica (GIS, siglas en inglés), particularmente MapInfo.
Fichero con la estructura del campo dividido en celdas y con la información pertinente a cada una: localización (latitud, longitud), rendimiento. El campo estará dividido en celdas pequeñas (en unidades de metros cuadrados).

Las siguientes figuras muestran el proceso de obtención de un MR y cómo influye en el desarrollo de la aplicación de Dosis Variables (DV) (Figura 2 y Figura 3). 


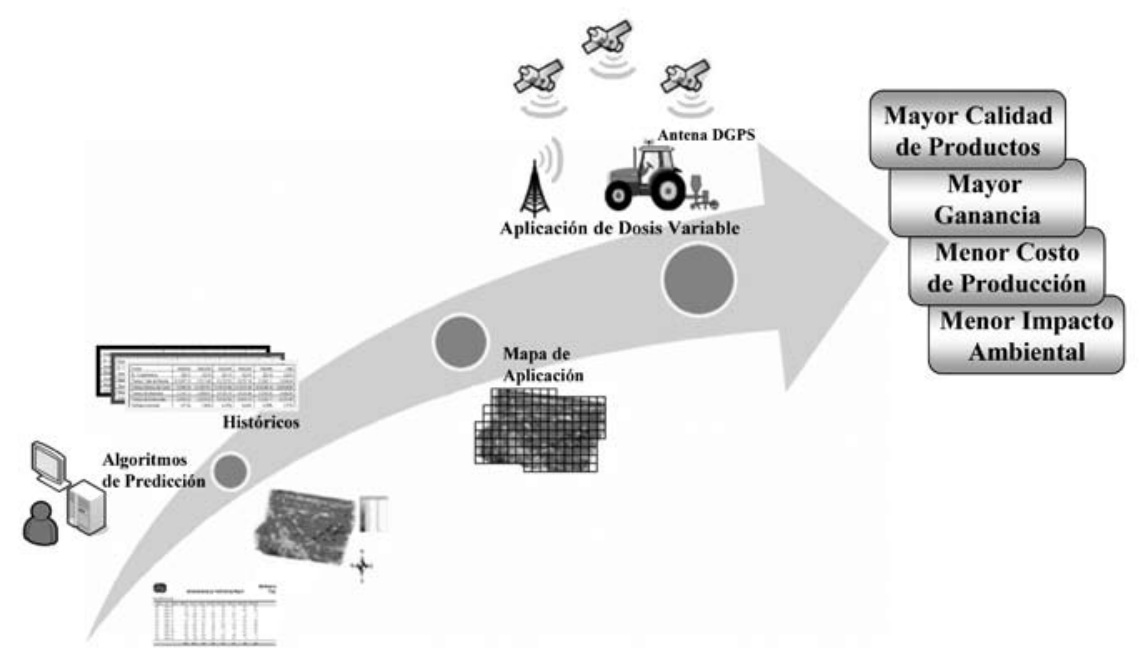

Figura 3. Aplicación de Dosis Variable.

\subsection{Descripción de la Aplicación Propuesta}

Algunas de las principales funcionalidades son descritas a continuación:

- Calcular Rendimiento: Con esta opción los puntos de rendimiento son calculados y actualizados en la Base de Datos (BD).
- Crear el Mapa de Rendimiento: Se crea una imagen y se visualiza en la pantalla, donde será acompañada de una escala que representará el valor del rendimiento.

A continuación se muestra una imagen de la pantalla principal del sistema (Figura 4) y una imagen de la pantalla de configuración del algoritmo para obtener el rendimiento puntual y visualización del Mapa de Rendimiento (Figura 5).

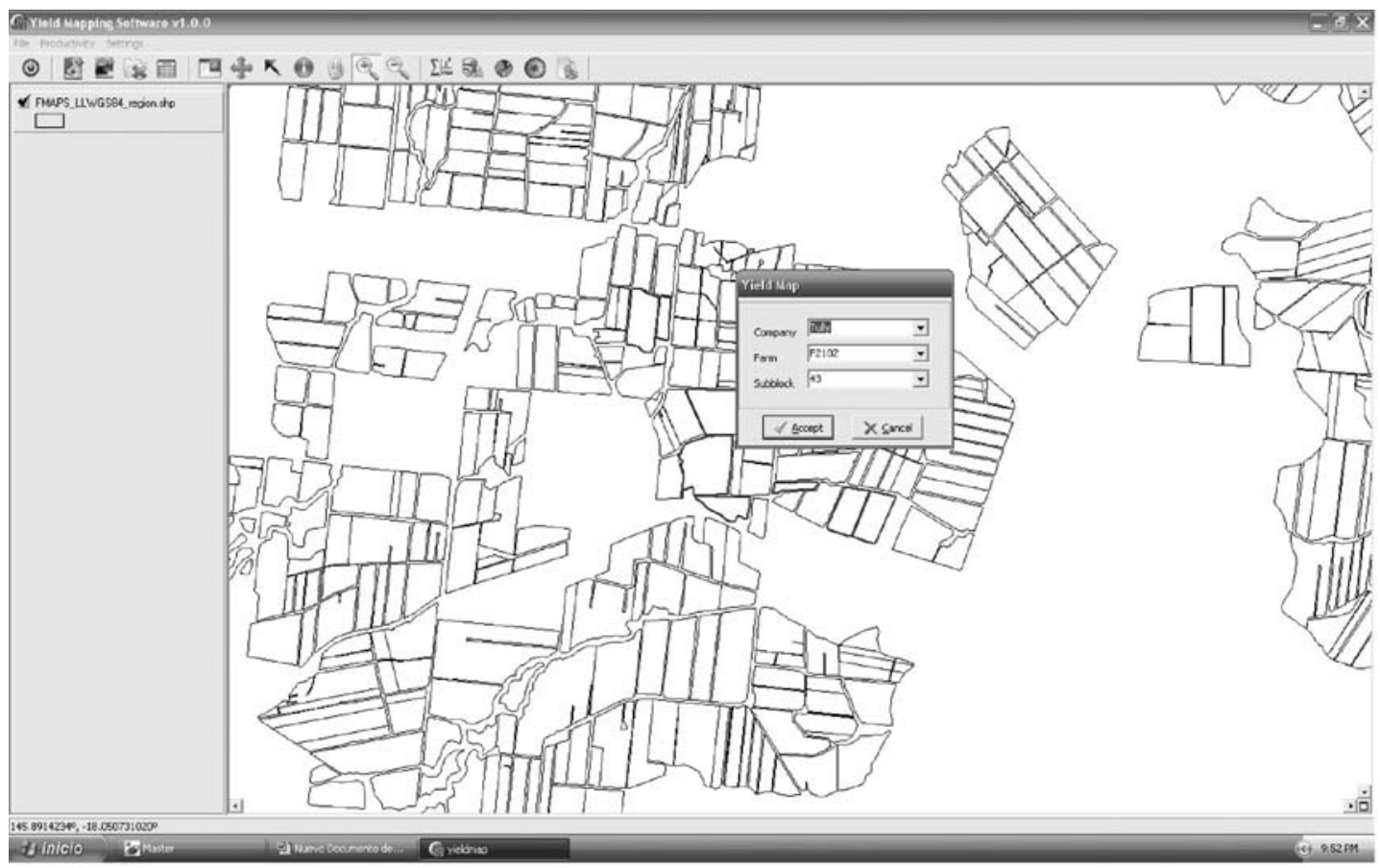

Figura 4. Pantalla principal del Sistema para la Generación de los Mapas de Rendimiento. 


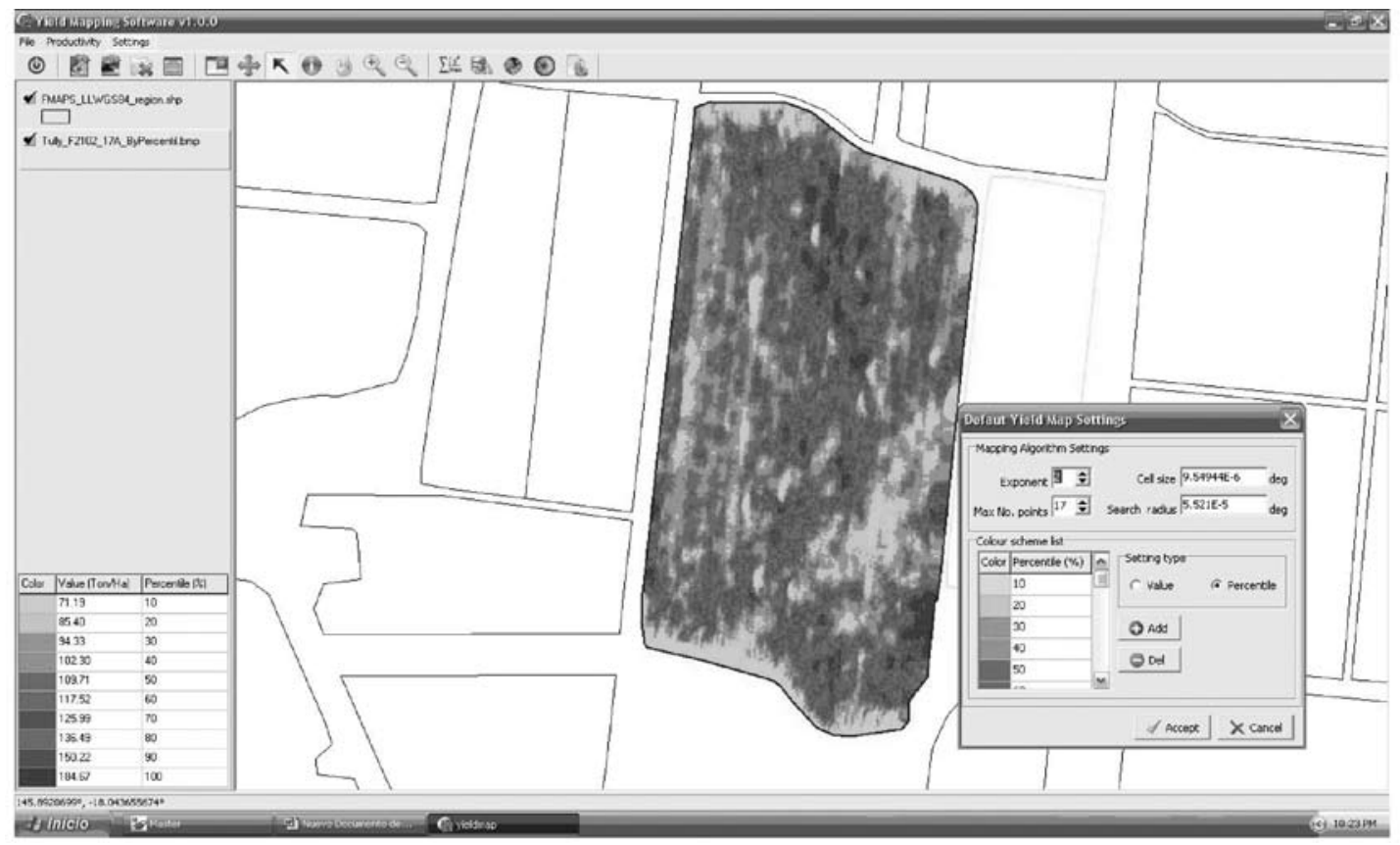

Figura 5. Pantalla de configuración del algoritmo para obtener el rendimiento puntual y visualización del Mapa de Rendimiento.

\section{Prueba realizada en el año 2007, distrito de Herbert, Australia}

En noviembre de 2007 se realizó una prueba completa del sistema, iniciada por especialistas del HCPSL, AgGuide (empresa australiana líder en el desarrollo de tecnologías para la AP) y técnicos de TechAgro. La prueba consistía en generar un MR con el sistema de TechAgro y otro con el sistema de AgGuide y compararlos para ver cual de los dos se acercaba más a la distribución real del rendimiento en el cultivo. El campo seleccionado fue cortado de forma experimental, y fueron comparados los resultados generados por el sistema con los obtenidos de forma manual.

El sistema de AgGuide contaba sólo con un monitor de rendimiento (sensor de flujo de caña y OBC) cuya salida era un fichero Latitud, Longitud, Rendimiento, pero faltaba un sistema para generar los MR, como consecuencia necesitaban utilizar un GIS de propósito general, ArcView, para generar las imágenes georreferenciadas, implicando realizar de forma manual todos los pasos para generar un MR, lo que se convertía en un proceso lento y tedioso.
El sistema de TechAgro contaba con un monitor de rendimiento, el OBC y el Yield Mappin Software, que generaba como salida una imagen georreferenciada correspondiente al mapa de rendimiento (Figura 5).

El campo donde se desarrolló la prueba fue dividido en tres secciones continuas verticalmente y en 10 horizontales quedando celdas de cuatro surcos o carriles, de un ancho de 1,4 metros (m) cada uno y $12 \mathrm{~m}$ de largo aproximadamente. Esto generaba 30 celdas de $5,6 \mathrm{~m}$ de ancho por $12 \mathrm{~m}$ de largo.

En la prueba cada vez que la cosechadora cortaba y llegaba al final de la celda (la cual estaba señalizada en el campo), esta se detenía y se pesaba la caña cortada. Para realizar el pesaje de la caña cortada se utilizó un tractor con una carreta que tenía adaptada una pesa hidráulica. Los datos obtenidos de forma manual (Figura 6) eran registrados por los especialistas [10]. Al finalizar el corte del campo, se generaban los respectivos MR de TechAgro y AgGuide, los que pasarían por un proceso de revisión y comprobación con los registros manuales. Estos datos tenían la siguiente estructura: 


\begin{tabular}{|c|c|c|c|c|c|c|c|c|c|c|c|c|c|}
\hline \multicolumn{3}{|c|}{$6 \mathrm{M}$ buffer row } & \multicolumn{4}{|c|}{$6 \mathrm{M}$ buffer row } & \multicolumn{4}{|c|}{$6 \mathrm{M}$ buffer row } & \multirow[b]{2}{*}{97} & \multirow[t]{2}{*}{10777} & \multirow[t]{2}{*}{8965} \\
\hline 70 & 57 & 32 & 68 & 77 & 65 & 86 & 46 & 42 & 112 & 83 & & & \\
\hline 227 & & & & 274 & & & & 334 & & & & 835 & \\
\hline $1 \_10$ & & & & 5_10 & & & & 9_10 & & & & & \\
\hline$\overline{39}$ & 19 & 55 & 93 & -55 & 139 & 86 & 68 & 26 & 19 & 52 & 120 & & \\
\hline 206 & & & & 348 & & & & 217 & & & & 771 & \\
\hline 1 9 & & & & 5_9 & & & & 9 & & & & & \\
\hline 1 & 20 & 0 & 0 & 161 & 30 & 144 & 45 & 33 & 44 & 80 & 150 & & \\
\hline 21 & & & & 380 & & & & 307 & & & & 708 & \\
\hline 1_8 & & & & 5_8 & & & & 9 & & & & & \\
\hline$\overline{4} 7$ & 40 & 45 & 169 & $\overline{177}$ & 30 & 104 & 50 & 81 & 73 & 92 & 69 & & \\
\hline 301 & & & & 361 & & & & 315 & & & & 977 & \\
\hline 1$] 7$ & & & & $5 \_7$ & & & & $9 \_7$ & & & & & \\
\hline$\overline{6} 1$ & 36 & 54 & 63 & 38 & 60 & 77 & 51 & 81 & 73 & 92 & 69 & & \\
\hline 214 & & & & 226 & & & & 315 & & & & 755 & \\
\hline $1 \_6$ & & & & $5 \_6$ & & & & $9 \_6$ & & & & & \\
\hline 59 & 44 & 101 & 64 & 75 & 103 & 93 & 136 & 55 & 127 & 117 & 86 & & \\
\hline 268 & & & & 407 & & & & 385 & & & & 1060 & \\
\hline 15 & & & & 55 & & & & $9 \_5$ & & & & & \\
\hline 131 & 48 & 129 & 67 & 137 & 53 & 108 & yy & -1 & 6 & 8 & 2 & & \\
\hline 375 & & & & 397 & & & & 17 & & & & 789 & \\
\hline 1_4 & & & & $5 \_4$ & & & & 9_4 & & & & & \\
\hline$\overline{5} 2$ & 38 & 59 & 73 & -50 & 85 & 99 & 108 & 79 & 78 & 220 & 92 & & \\
\hline 222 & & & & 342 & & & & 469 & & & & 1033 & \\
\hline 13 & & & & 5_3 & & & & $9 \_3$ & & & & & \\
\hline 89 & 67 & 145 & 98 & 38 & 112 & 101 & 49 & 104 & 42 & 152 & 113 & & \\
\hline 399 & & & & 300 & & & & 411 & & & & 1110 & \\
\hline 1_2 & & & & 5_2 & & & & $9 \_2$ & & & & & \\
\hline$\overline{95}$ & 37 & 58 & 40 & $\overline{112}$ & 116 & 81 & 31 & 92 & 38 & 132 & 55 & & \\
\hline 230 & & & & 340 & & & & 317 & & & & 887 & \\
\hline 1 1_1 & & & & 5_1 & & & & 9 & & & & & \\
\hline$\overline{46}$ & 52 & 58 & 84 & 34 & 114 & 88 & 55 & 86 & 73 & 72 & 113 & & \\
\hline 240 & & & & 291 & & & & 344 & & & & 875 & \\
\hline $13 m$ & buff & er zor & & $13 \mathrm{mb}$ & ouffer & zon & & $13 m$ & uffer $z$ & & & & \\
\hline 96 & 32 & 77 & 78 & 90 & 89 & 99 & 69 & 45 & 84 & 121 & 97 & & \\
\hline 283 & & & & 347 & & & & 347 & & & & 977 & \\
\hline
\end{tabular}

Figura 6. Representación de los datos registrados de forma manual por los especialistas.

Para explicar la estructura de esta tabla, se ha señalizado, enmarcada por un rectángulo, una sección que se describe a continuación:

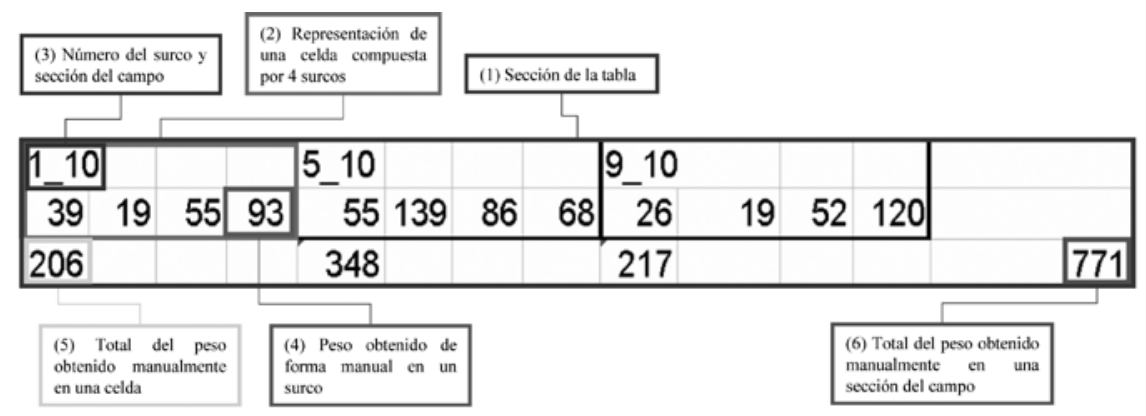

Figura 7. Descripción de la sección de la tabla con los datos registrados manualmente. 
Es la sección de la tabla tomada de la Figura 7 para explicar su estructura.

1. Representa una celda de cuatro surcos que fue georreferenciada, donde se registró el peso obtenido de cada surco que fue analizado.

2. Identificador único de la celda dentro del campo.

3. Peso registrado del surco en cuestión (el valor 39 pertenece al primer surco de la celda, el 19 pertenece al segundo surco y así sucesivamente).
4. Peso total de la celda, no es más que la suma total de los pesos de los cuatro surcos perteneciente a la celda.

5. Es el peso total de la sección del campo, la suma de los pesos obtenidos en cada una de las celdas.

\section{Resultados}

Después de terminar el corte del campo completo, el siguiente paso de la prueba era generar el mapa de rendimiento por ambos monitores.

El mapa de rendimiento generado con el sistema de TechAgro fue el siguiente:

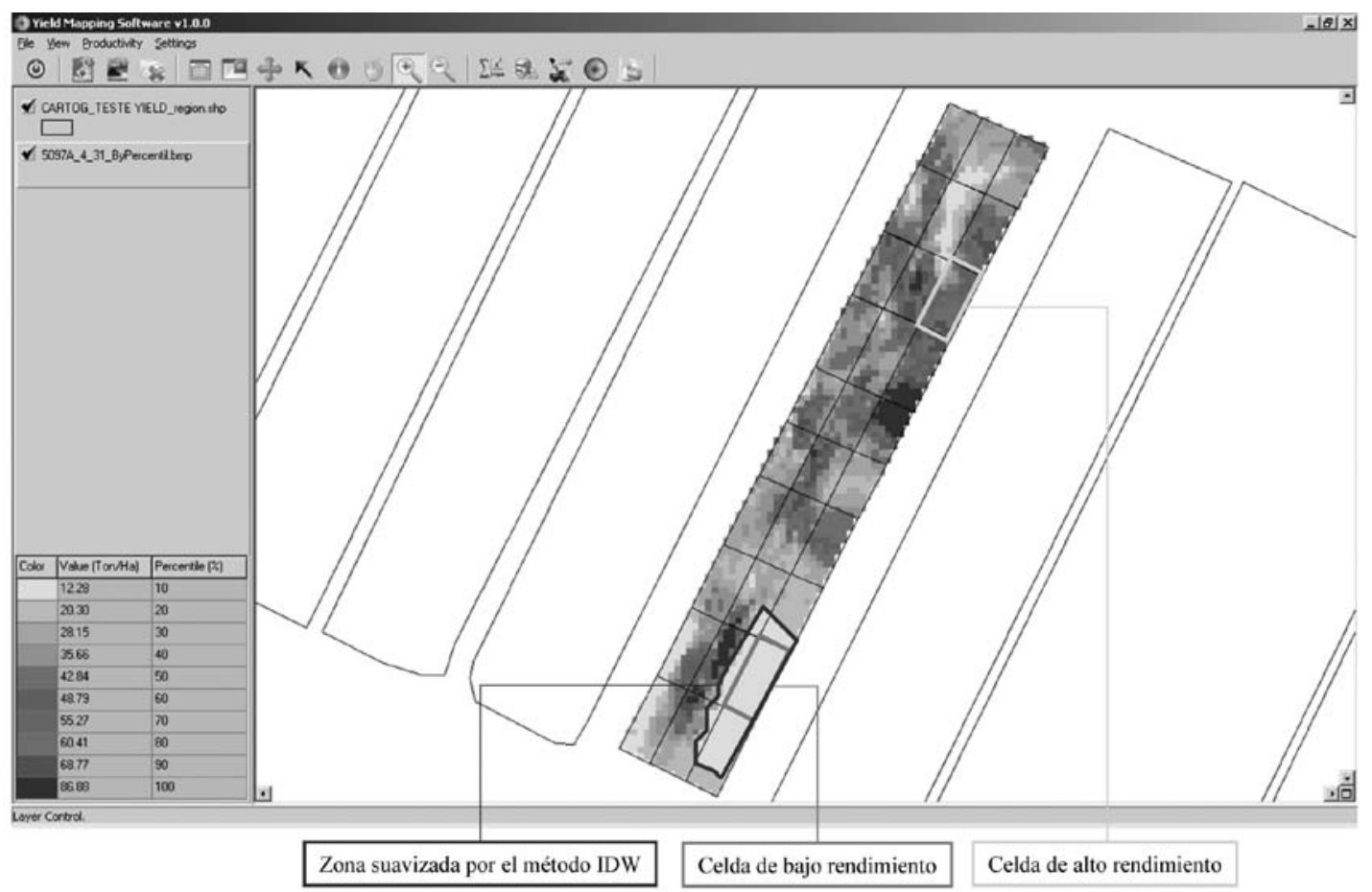

Figura 8. MR generado por el sistema en la prueba de Herbert.

En la figura anterior (Figura 8) se observa que la celda 1_9 (enmarcada por un rectángulo, observar la tabla mostrada en la Figura 7) tiene un peso extremadamente bajo, debido a que en esa localización no había caña, pues fue cortada manualmente por los especialistas para comprobar que el algoritmo del cálculo del rendimiento asignaba un valor bajo de rendimiento en los lugares donde había poca caña, mostrado con un color de tonalidad claro, lo cual se correspondió con el mapa generado por el sistema. La zona del mapa encerrada en un polígono cerrado, representa la aplicación del método de interpolación IDW y como consecuencia se aplica a esa zona un color claro, indicando que existe una zona de bajo rendimiento [11].

La celda 1_3 (enmarcada por un rectángulo) tiene un rendimiento promedio de $68 \mathrm{t} / \mathrm{ha}$, lo que corresponde representar el color en tonalidades fuertes.

El sistema de AgGuide en esta prueba logró generar un fichero de Latitud, Longitud, Rendimiento, pero el cálculo de la variable rendimiento presentaba 
un problema: no tiene en cuenta el peso real de la caña cosechada en el campo, por lo que no es posible calibrar ese peso con los puntos registrados por el OBC. Además, fue incapaz de generar un MR, pues los rendimientos calculados a los puntos registrados presentaban un margen de error muy grande [11].

Al finalizar la prueba, los especialistas australianos consideraron al sistema como el único capaz de generar un MR lo más cercano posible a la realidad en el campo. La empresa AgGuide aceptó la verdad de poseer un error tecnológico en su algoritmo y reconoció la calidad de los MR generados por el sistema [11]. A partir de conocerse los resultados de esa prueba, la empresa AgGuide dejó de comercializar los monitores de rendimientos desarrollados por ellos hasta ese momento, reconociendo el desempeño del sistema para generar los MR.

Los resultados han sido documentados de manera oficial por los especialistas australianos Lawrence Dibella y Mike Sefton, del grupo HCPSL mencionado anteriormente, en el trabajo "A Comparison of Yield Mapping System in the Herbert Sugar Industry" que será publicado por primera vez en el Evento Internacional Geomática 2009, perteneciente a la Convención Internacional de Informática 2009. Varios artículos de la prensa local en el distrito de Herbert han referenciado el avance de la AP en esa región, gracias a la adquisición de tecnologías cubanas como el generador de mapas de rendimiento (Figura 8), el SGMA y el OBC de TechAgro (AGM-200A) para el cultivo de la caña de azúcar, donde argumentan la posibilidad de continuar las investigaciones en áreas dentro de la AP como la ADV y el seguimiento Online de las maquinarias agrícolas, así como de los medios de transportes de caña, entre ellos los trenes. Esto permitiría, obtener en tiempo real, el rendimiento estimado de un campo mientras se está cosechando [12], además de poder gestionar la logística del movimiento de caña desde los campos hacia el central.

Otras pruebas han sido realizadas en Brasil por la empresa TechAgro, pero sus resultados han estado enfocados en el desarrollo de un monitor de rendimiento más preciso y con un mejor diseño para incorporar a la cosechadora. Muchas de las empresas que fabrican cosechadora están insertando a sus producciones monitores de rendimiento que son adaptables a la mayoría de los OBC más usados en la producción de azúcar. En un futuro, esto implicaría el desarrollo de un OBC que sea adaptable a los sensores de rendimiento desarrollados por las fábricas, pero el sistema continuaría funcionando de la misma forma, sin ningún tipo de cambio.

\section{Conclusiones}

Mediante el uso de las tecnologías del GPS, se desarrolló una aplicación para la generación de Mapas de Rendimiento de forma fácil y sencilla. Es alcanzable para cualquier usuario la obtención de un MR sin tener conocimientos previos de los algoritmos matemáticos a usar. Se obtuvo una herramienta de las primeras de su tipo, pues en el mercado todavía no existen desarrollos similares. Esta herramienta es un desarrollo integrado al SGMA y ayuda a la toma de decisiones para la próxima cosecha. Anexo a la imagen que es una de las salidas de esta aplicación, también se obtiene un fichero con una descripción del campo en cuanto a rejillas o celdas que apoyado en ciertas reglas basadas en la composición de los suelos se puede usar para la aplicación de DV en la fertilización de los cultivos. Actualmente no existen estándares que establezcan los formatos de estos datos, por lo que queda abierto al ajuste del usuario obtener el formato del fichero de entrada para la DV.

\section{Literatura Citada}

[1] Zhang, N., M. Wang, et al.

2002 Precision agriculture - a worldwide overview. Computers and Electronics in Agriculture, 36 (2-3): 113-132.

[2] Romans, W., B. Poore, et al.

2000 Advanced instrumentation for agricultural equipment. Instrumentation \& Measurement Magazine, IEEE 3 (1): 26-29.

[3] Vázquez, A.

$2004 \mathrm{La}$ agricultura de precisión se extiende en Cuba. El Habanero.
[4] Gil, E.

2008 Situación actual y posibilidades de la Agricultura de Precisión, Escuela Superior de Agricultura de Barcelona, 18 de febrero 2002.

[5] Bongiovanni, R.

2001 VII Congreso Nacional del Maíz. AIANBA, Pergamino, Argentina.

[6] Auernhammer, $\mathrm{H}$.

2001 Precision farming - the environmental challenge. Computers and Electronics in Agriculture 30 (1-3): 31-43. 
[7] Pérez, F., Zamora, M., Vicini, L., Monasterio, M. 2002 AgroVisión, vol. 38.

[8] Cadenas, A.

2000 Escuela Técnica Superior de Ingenieros Industriales y de Ingenieros de Telecomunicación de Bilbao, Universidad del País Vasco.

[9] Bragachini, M., Méndez, A.

2004 Tecnología Disponible para Aplicaciones de Insumos Sitio Específico.

[10]Lago, C.

2008 Generación del Mapa de Rendimiento y Reporte de Productividad del cultivo de la caña de azúcar utilizando tecnología GPS. Centro de Estudios de Ingeniería y Sistemas (CEIS). Facultad de Ingeniería Informática, Instituto Superior Politécnico "José Antonio Echeverría". La Habana. MSc., 103.

[11]Dibella, L.P., A.W.W., Sefton, M., Esquivel, M., Fernández, F., Kerkwyk, R.E., Delai, R., Schroeder, B. L.

2008 BSES Limited, Herbert, Townville, Queensland, Australia.

[12] Kerr, B.

2008 Good Farming Practices, pp. 12, 13. 
